\title{
Stem Cells for Huntington's Disease (SC4HD): An International Consortium to Facilitate Stem Cell-Based Therapy for Huntington's Disease
}

\author{
The SC4HD Consortium ${ }^{1, *}$
}

Pre-press 26 February 2021

\begin{abstract}
Huntington's disease (HD) research is entering an exciting phase, with new approaches such as huntingtin lowering strategies and cell therapies on the horizon. Technological advances to direct the differentiation of stem cells to desired neural types have opened new strategies for restoring damaged neuronal circuits in HD. However, challenges remain in the implementation of cell therapy approaches for patients suffering from HD. Cell therapies, together with other invasive approaches including allele specific oligonucleotides (ASOs) and viral delivery of huntingtin-lowering agents, require direct delivery of the therapeutic agents locally into the brain or cerebrospinal fluid. Delivering substances directly into the brain is complex and presents multiple challenges, including those related to regulatory requirements, safety and efficacy, surgical instrumentation, trial design, patient profiles, and selection of suitable and sensitive primary and secondary outcomes. In addition, production of clinical grade cell-based medicinal products also requires adherence to regulatory standards with extensive quality control of the protocols and cell products across different laboratories and production centers. Currently, there is no consensus on how best to address these challenges. Here we describe the formation of Stem Cells For Huntington's Disease (SC4HD: https://www.sc4hd.org/), a network of researchers and clinicians working to develop guidance and greater standardization for the HD field for stem cell based transplantation therapy for HD with a mission to work to develop criteria and guidance for development of a neural intra-cerebral stem cell-based therapy for $H D$.
\end{abstract}

Keywords: Neuroscience, Huntington's disease, stem cells, transplantation

\section{BACKGROUND}

Huntington's disease (HD) is an inherited autosomal dominant neurodegenerative condition caused by a CAG repeat in the huntingtin gene on chromosome

\footnotetext{
${ }^{1}$ A full list of collaborating members and affiliations appears at the end of the paper. Collaborators are mentioned in alphabetical order.

*Correspondence to: jmcanals@ub.edu, RosserAE@ cardiff.ac. uk, and lmthomps@uci.edu.
}

4. Onset is variable over ages ranging from childhood to older age groups, but most commonly in mid-life, resulting in slowly progressive deterioration of motor, cognitive and behavioural functions, in addition to a range of currently less well-defined symptoms. The disease progresses over a period of 1-3 decades, depending on time of diagnosis, with most sufferers eventually requiring help with all activities of daily living. Although some aspects of HD, notably chorea and a subset of the behavioural symptoms 
such as depression and anxiety, may respond to a variable extent to existing conventional treatments, most symptoms are not treatable and there are no disease-modifying treatments currently available.

The gene mutation in HD is known to lead to progressive central nervous system dysfunction and neurodegeneration, with striatal degeneration being an early and obvious, though not exclusive, consequence. Most experimental cell therapy to date has focused on the striatum with the aim being to replace the striatal neurons lost to the disease process in order to effect circuit repair and/or to increase the survival of the remaining neuronal and glial striatal cells. In addition, targeting of other brain regions has been entertained and will become increasingly important as the technology advances, given that HD eventually affects much of the brain. To date, transplantation of a variety of cells into both excitotoxic and genetic rodent models of $\mathrm{HD}$, as well as into large animal models of excitotoxicity, has supported the notion that cells can survive transplantation into the brain for extended periods of time and lead to functional improvement. Transplantation of human fetal striatal cells in humans has also demonstrated long term graft survival and safety, although the conditions for evaluating potential efficacy on a consistent basis are not yet defined. Thus, cell therapy for HD is in its early stages of clinical translation.

However, given 1) the existence of early translational studies for human fetal cell transplantation in HD supporting safety, 2) the publication of protocols for striatal neuronal and glial progenitor differentiation from human pluripotent stem cells, and 3) the published preclinical efficacy data following transplantation of precursor cells and glial progenitor cells in HD animal models, we consider it timely to generate a collaborative network, SC4HD, to guide the establishment of first-in-human advanced therapy medicinal product (ATMP) cell transplantation studies for HD. The network brings together experts in stem cell biology, cell transplantation, clinical grade cell production, neurosurgery, and clinical evaluation to accelerate the safe clinical translation of stem cell therapy and to deliver comprehensive standardization and guidance for the field, spanning the full range of therapeutic considerations as described in detail below. Developing these criteria will involve consideration of all the elements required to undertake such trials in HD.

SC4HD will convene regular meetings with constituents and others with relevant expertise, including patients or patient advocates. We will also keep

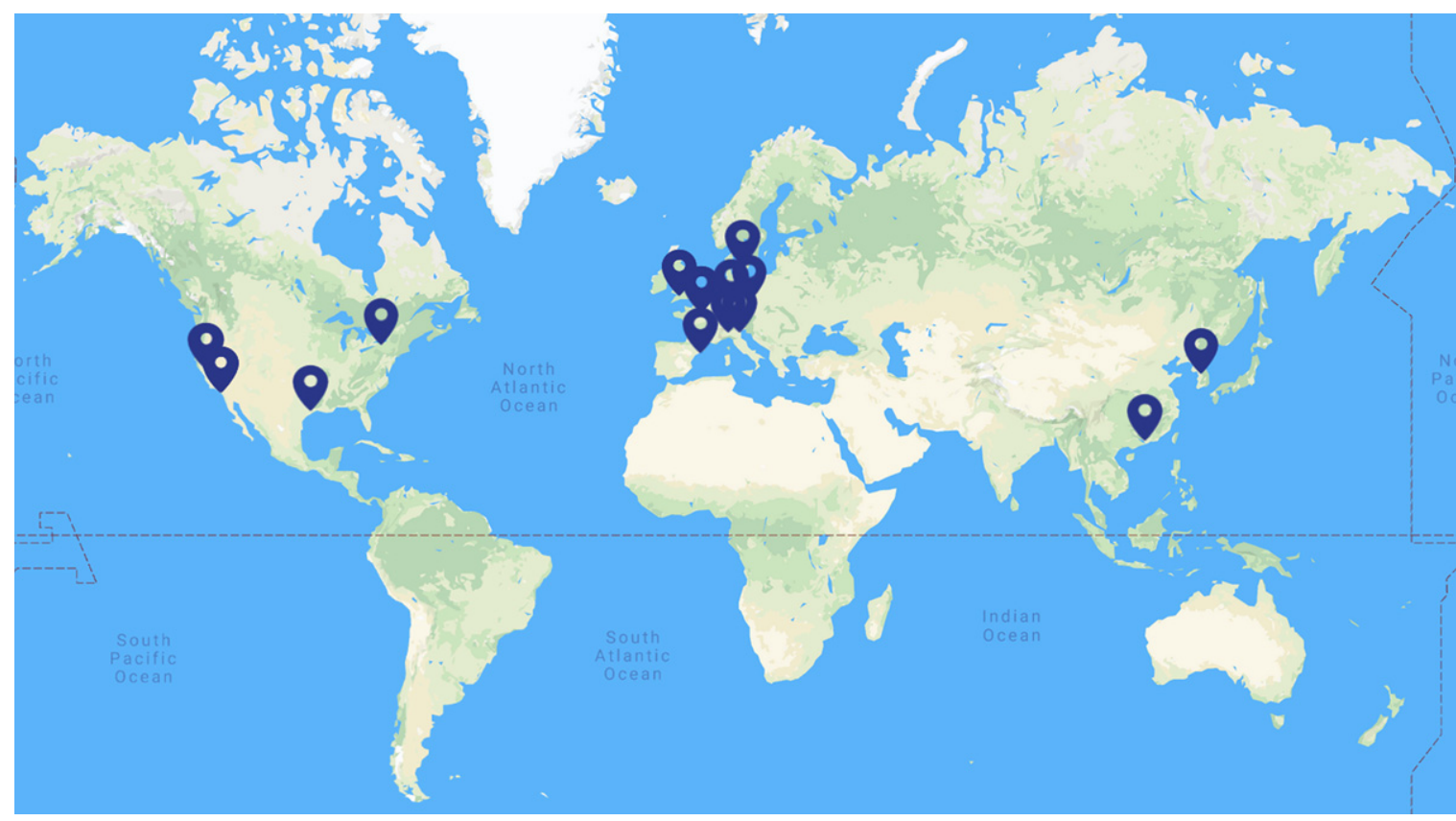

Fig. 1. The current geographical distribution of the members involved in SC4HD consortium. 28 members are distributed on 10 countries from 3 continents. 
abreast of existing and emerging therapeutic approaches in order to continually reappraise the likely and appropriate role of cell therapies in the treatment of HD. While stem cell therapies for HD have their unique challenges and opportunities, we have and will continue to seek guidance and learn from shared experiences across other therapeutic modalities, such as those using small molecules and ASOs, genome editing, and gene therapy. We will integrate emerging knowledge from ongoing or planned stem cell transplantation trials for other disease indications, including Parkinson's disease (PD) and amyotrophic lateral sclerosis (ALS), and from lessons learned through G-force and other similar consortia advancing translational research in other neurodegenerative diseases. We have benefitted from the guidance of participants and advisors from related disciplines and organizations including the California Institute for Regenerative Medicine (https://www. cirm.ca.gov/), European Huntington's Disease Network (http://www.ehdn.org/), and CHDI Foundation (https://chdifoundation.org/).

\section{ORIGINS OF CONSORTIUM AND GEOGRAPHIC DISTRIBUTION}

\section{May 2018 inaugural meeting at the University of California: Overview}

The inaugural meeting on May 1-2, 2018 of SC4HD was a successful and exciting event cosponsored by the California Institute for Regenerative Medicine (CIRM) and CHDI Foundation. The meeting aimed to build upon efforts by CIRM, Repair $\mathrm{HD}$, and other entities to help bring stem cells safely to the clinic for HD. We included individuals who have used, or are using, stem cell based therapeutic approaches for HD, PD, ALS, and spinal cord injury. Bringing together experts working in many areas relevant to this mission allowed sharing of knowledge and experience, leading to greater coherence across specialty and geographical boundaries to achieve preclinical criteria and accelerate progress to the clinic. It was recognised that in order to clear the roadblocks to allow cell-based therapies to advance and to prevent delays in the design of well-defined clinical trials for stem cell approaches, it will be necessary to tackle preclinical and translational issues in parallel and to bring together expertise across many disciplines, underlining the motivation to build an international consortium.
We hosted 56 attendees in total with 6 attendees joining us by web conference for the two-day event at the Beckman Center for National Academies of Science, Engineering and Medicine and UC Irvine Stem Cell Research Center. The first day of the meeting began with an overview of HD stem cell efforts globally, followed by discussions on a) manufacturing and cell products, b) preclinical criteria, c) clinical development - immunological issues, surgery and devices, d) brain imaging/human trial design, and e) regulation and ethics. The second day of the meeting focused on next steps, groups aspirations, program management, development and strategy of the network, potential future funding sources and venue for the next meeting.

\section{9 meeting in Barcelona, Spain: overview and additional engagement with EHDN}

A second meeting took place in Barcelona in April 2019, this time as part of a meeting of the EHDN Advanced Therapies Working group. The aims of the Advanced Therapies Working group overlap extensively with SC4HD, but are broader, extending to delivery of fluid substances, such as for gene therapies or antibodies. The outcome of the broader group meeting can be found on the EHDN website (http: //www.ehdn.org/es/advanced-therapies-wg/). During this second meeting, the SC4HD subgroup focused on the strategy to form the network and discussed how to delineate a charter,an overarching framework, and a pipeline to actively develop guidance. We identified opportunities for alignment with organizations such as ISSCR and other disease-focused networks (e.g., G-force for PD) for regulatory engagement, general preclinical guidelines, surgical considerations and defined attributes for cell manufacturing.

A charter group of members was formed and a discussion paper planned to build on the knowledge gained since the first HD fetal cell transplant paper 20 years ago [1]. This meeting, the aims of SC4HD were consolidated, several goals and focus areas were identified, as listed below, and work has begun on the production of reports and to set up relevant task forces and working groups.

\section{THE GOALS OF THE CONSORTIUM AND CHALLENGES}

The overall goal of the SC4HD consortium is to define the challenges to be addressed across the spectrum of cell manufacturing, preclinical assessments 


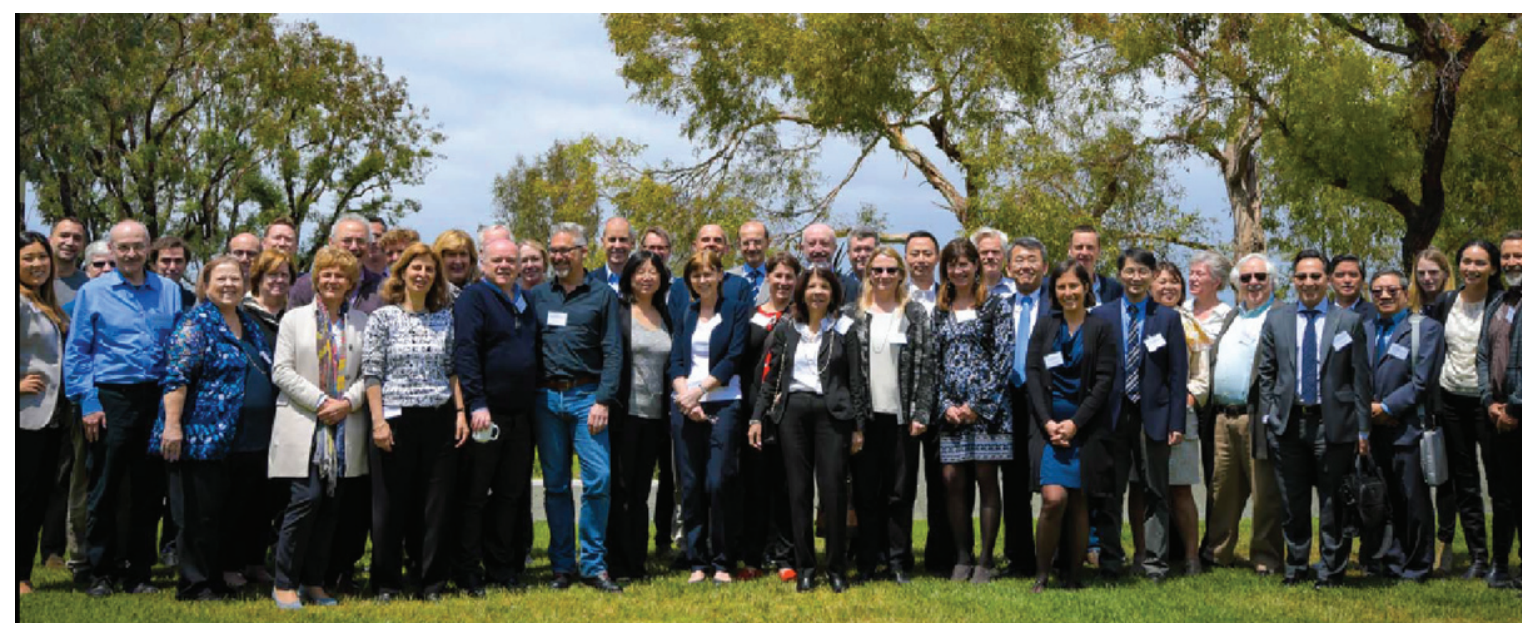

Fig. 2. Picture of the 50 face to face attendees of the first SC4HD meeting that took place at the Beckman Center for National Academies of Science, Engineering and Medicine and UC Irvine Stem Cell Research Center.

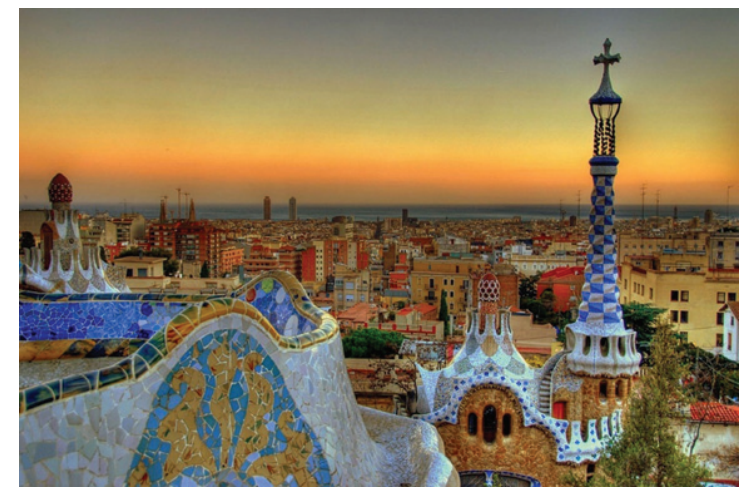

Fig. 3. 26 researchers attended the Barcelona meeting that took place at the NH Collection Podium Hotel. The meeting was a joint effort of the SC4HD consortium and the Advanced Therapies Working group of the EHDN.

and clinical application, to enable the promise of stem cell-based therapies to be transplanted in the brain for treatment of HD. We seek to address these challenges and to establish guidelines for preclinical and clinical development of stem cell based therapies. We recognise that this is likely to require an iterative approach with bidirectional flow of information between preclinical and clinical activities. As a first step, a white paper is being developed in which we will identify and provide detailed discussion of the key challenges regarding either neuroprotection, circuitry restoration or a combination of the two. The challenges include: a. Defining the principles to guide decisions to move a potential cell-based therapy from basic research towards a first-in-human trial. Key challenges include identification of the proposed mechanism of biological activity of the investigational cellular product to achieve neurorestorative effects and the optimal preclinical models for assessing efficacy. It is critical to establish key readouts that define the preclinical data sufficient to consider the candidate as a serious therapeutic medicinal product. These include the choice of cells and strategies, the types and nature of preclinical models, requirements for potential immunosuppression, safety toxicology and tumorigenicity studies and the readouts that are relevant and predictive of outcomes in human HD.

b. Optimization of cell product for HD therapy. Optimization of the cell product is dependent on the therapeutic strategy (neuroprotection, neuroregeneration and/or circuit restoration). Efforts using neural (neuronal or glial) progenitors to restore striatal cell populations have shown some success in experimental animals. Human embryonic or induced pluripotent stem cells can be guided to differentiate into neural or glial progenitors at different stages with a range of potential differentiation capacities. The differentiation process must be optimised to ensure that cell products can mature into cell populations with the desired characteristcs and the range of differentiated progeny needs to be characterised. Optimized protocols need to be GMP standardized to define 
the resultant cellular product in terms established both before and after transition to GMP manufacturing.

c. Cell manufacturing and scale-up for human use. Compliance with Good Manufacturing Practice (GMP) is mandatory for ATMPs for delivery into man; basic research procedures must be translated into a GMP quality system, implementing quality control and quality assurance and avoiding or minimizing reagents of animal origin. Manufacturing process must also establish the quality attributes of the final product such as cell number, viability, dose, phenotype, karyotype and copy number variation and genomic stability, among others.

Preclinical assessment of safety and efficacy studies may also require the use of large animal models with larger brain sizes and similar anatomical organization to that of humans. Depending on the purpose of their use, such models may also model elements of HD pathology. Potential uses of large animal models include the definition of follow up techniques to characterise the precision and safety of neurosurgical delivery, the viability and distribution of the cellular product, to scaleup from rodents and determine efficacious dose, and to guide the design of neurosurgical delivery devices for first-in-human trials (see below).

d. Optimal delivery of cells to the brain. The structural and biological complexity of the brain requires optimization of neurosurgical devices and procedures. To date, there are no CE marked devices that have been optimised for cell delivery and although neurosurgical approaches for delivery of cells into the brain is described in the literature, the procedures have not been defined or explored in detail.

e. Implementation of clinical trials in practice: Clinical trial design. In clinical studies a major challenge is conforming to regulatory standards at the same time as ensuring efficient design principles. In the EU, clinical trials are mandatory for testing cell based therapies (ATMPs). Similarly, in the US, an Investigational New Drug Application (IND) request is submitted to the FDA for authorization to administer an investigational cellular therapy product for human clinical trials. The use of placebo controlled designs is challenging in this area, especially for early stage studies, however, its consideration is an important component of the rigorous evaluation of new therapies, both to account for the psychological expectation of effectiveness and neurobiological effect of the intervention.

Patient selection criteria. Patient selection criteria for cellular therapy trials requires consideration of disease-stage, phenotypic variability, the presence of extrastriatal pathology and striatal volume, the latter influencing the safety of targeting and delivery of cellular products. The target population will likely include those with early or mid-stage manifest HD for whom robust outcome measures have been validated. The determination of disease severity for a cellular therapy trial should be based on both the therapeutic mechanism(s) of the cellular therapeutic in the targeted patient population and on the safety and precision of neurosurgical delivery.

Defining post transplantation management including immunosuppression or stem cell lines selection or genetic engineering to maximise graft survival and integration, graft-host interactions and the importance of long term follow-up to measure repair beyond replacement. In addition, it is important to define the long term follow-up in trials for cell transplantation, which includes not only to measure clinical outcomes but also for pharmacovigilance of the patients treated with cell-based product.

\section{SUMMARY}

There is an exciting opportunity to apply stem cell-based therapies in HD. At the same time, significant challenges remain and these new therapeutic approaches will benefit from insight from different areas of expertise. The goal of SC4HD investigators is to work together with the HD community to develop criteria and guidance and address these challenges to rapidly and safely bring potentially beneficial neural intra-cerebral stem cell-based therapies to patients with this devastating disease. Ongoing information about future meetings and updates will be provided on the SC4HD site.

\section{ACKNOWLEDGMENTS}

We thank the California Institute of Regenerative Medicine (CIRM), European Huntington's Disease Network (EHDN), and CHDI Foundation for support of the reported meetings. We also thank Yuna Muyshondt for all her assistance with organizing network communication. 


\section{CONFLICT OF INTEREST}

The authors have no conflict of interest to report.

\section{REFERENCES}

[1] Bachoud-Levi AC, Remy P, Nguyen JP, Brugieres P, Lefaucheur JP, Bourdet C, et al. Motor and cognitive improvements in patients with Huntington's disease after neural transplantation. Lancet. 2000;356:1975-9.

The complete list of SC4HD Consortium authors is as follows, in alphabetical order: Romina Aron Badin $^{\mathrm{a}}$, Anne-Catherine Bachoud-Lévi ${ }^{\mathrm{b}}$, Gerhard Bauer ${ }^{\mathrm{c}}$, Monica Busse-Morris ${ }^{\mathrm{d}}$, Josep M. Canals ${ }^{\mathrm{e}}$, Philipp Capetian ${ }^{\mathrm{f}}$, Elena Cattaneo ${ }^{\mathrm{g}}$, Jefferson Chen $^{\mathrm{h}}$, Emanuele Cozzi ${ }^{\mathrm{i}}$, Zdenka Ellederova ${ }^{\mathrm{j}}$, Steven A. Goldman ${ }^{\mathrm{k}}$, William Gray ${ }^{\mathrm{l}}$, Liangxue Lai ${ }^{\mathrm{m}}$, Meng Li ${ }^{\mathrm{n}}$, Anna Morenkova ${ }^{\mathrm{o}}$, Guangjin Pan ${ }^{\mathrm{p}}$, Zhong Pei $^{\mathrm{q}}$, Unai Perpina Martin ${ }^{\mathrm{r}}$, Anselme Perrier ${ }^{\mathrm{s}}$, John C. Reidling ${ }^{\mathrm{t}}$, Anne E. Rosser ${ }^{\mathrm{u}}$, Jiwhan Song ${ }^{\mathrm{v}}$, Leslie M. Thompson ${ }^{\mathrm{w}}$ and Vicki Wheelock ${ }^{\mathrm{x}}$

${ }^{a}$ CEA, DRF, Institute of Biology François Jacob, Molecular Imaging Research Center (MIRCen), Fontenay-auxRoses, France. ${ }^{b}$ Department of Neurology, Henri Mondor Hospital, Interventional Neuropsychology, Institut Mondor de Recherche Biomédicale (IMRB, UPE) and at the Labex Institut de la Cognition (IEC, ENS), Créteil, France. ${ }^{\mathrm{c} I n s t i t u t e}$ for Regenerative Cures, University of California Davis, Sacramento, CA, USA. ${ }^{\mathrm{d}}$ Centre for Trial Research, School of Medicine, Cardiff University, Cardiff, United Kingdom. ${ }^{\mathrm{e}}$ Laboratory of Stem Cells and Regenerative Medicine, Department of Biomedical Sciences, Production and Validation Center of Advanced Therapies (Creatio), Faculty of Medicine and Health Sciences, Neuroscience Institute, University of Barcelona, Barcelona, Spain. ${ }^{\mathrm{f}}$ Department of Neurology, University Hospital Würzburg, Würzburg, Germany. ${ }^{\mathrm{g}}$ University of Milan and INGM - Istituto Nazionale di Genetica Molecolare Padiglione Invernizzi, Laboratory of Stem Cell Biology and Pharmacology of Neurodegenerative Diseases Department of Biosciences, Milan, Italy. ${ }^{\text {University }}$ of California, Irvine, School of Medicine - Neurosurgery, Orange, CA, USA. ${ }^{\mathrm{i}}$ Department of Cardiac, Thoracic and Vascular Sciences, Padua University Hospital - Ospedale Giustinianeo, Padova, Italy. ${ }^{j}$ Laboratory of Cell Regeneration and Plasticity, Institute of Animal Physiology and Genetics, Czech Academy of Sciences, Libechov, Czech Republic. ${ }^{k}$ University of Rochester, School of Medicine and Dentistry, and CTN Copenhagen, Rochester, NY, USA. ${ }^{1}$ Neuroscience and Mental Health Research Institute, School of Medicine, Cardiff University, University Hospital of Wales, Cardiff, United Kingdom. ${ }^{\mathrm{m}}$ Institute of Stem Cell Biology and Regenerative Medicine, Guangzhou Institutes of Biomedicine and Health, Chinese Academy of Sciences, Science Park, Guangzhou, China. ${ }^{n}$ Neuroscience and Mental Health Research Institute, Cardiff University, Cardiff, United Kingdom. ${ }^{\circ}$ University of California, Irvine, School of Medicine - Neurology, Irvine, CA, USA. ${ }^{\mathrm{p}}$ Guangzhou Institutes of Biomedicine and Health, Chinese Academy of Sciences, Science Park, Guangzhou, China. ${ }^{q}$ Department of Neurology, The First Affiliated Hospital, Sun Yat-Sen University, Guangzhou, China. ${ }^{r}$ Production and Validation Center of Advanced Therapies (Creatio), Faculty of Medicine and Health Sciences, University of Barcelona, Barcelona, Spain. ${ }^{\mathrm{s}}$ INSERM/UEVE/UMR 861, I-Stem, AFM - Institut of Stem Cell Therapy and Exploration of Monogenic Diseases, Evry, France. ${ }^{t}$ University of California, Irvine, Institute for Memory Impairments and Neurological Disorders, Irvine, CA, USA. ${ }^{\mathrm{u} C a r d i f f}$ University, Cardiff, UK. ${ }^{\mathrm{v}} \mathrm{CHA}$ Stem Cell Institute, Department of Biomedical Science, CHA University, Seongnam, Korea. ${ }^{\text {w}}$ University of California Irvine, Irvine, CA, USA. ${ }^{\mathrm{x}}$ Department of Neurology, University of California, Davis, Department of Neurology, Sacramento, CA, USA. 